\title{
Atom-Molecule Coherence Near a Feshbach Resonance in a Bose-Einstein Condensate
}

Sarah T. Thompson ${ }^{1}$, Neil R. Claussen ${ }^{1}$, Elizabeth A. Donley ${ }^{2}$, and Carl E. Wieman ${ }^{1}$

${ }^{1}$ JILA, National Institute of Standards and Technology and the University of Colorado, and the Department of Physics, University of Colorado, Boulder, Colorado 80309-0440

${ }^{2}$ National Institute of Standards and Technology, Boulder, Colorado

\begin{abstract}
Atom-molecule coherence in a Bose-Einstein condensate (BEC) has been demonstrated. Sudden changes were made to the magnetic field near a Feshbach resonance such that oscillations between atomic and molecular states were excited. The frequency of these oscillations was measured over a large range of magnetic fields and was found to be in excellent quantitative agreement with the predicted energy difference between two colliding atoms and the bound molecular state. This agreement indicates that we have created a quantum superposition of atoms and diatomic molecules, which are chemically different species.
\end{abstract}

\section{Introduction}

A major thrust in recent $\mathrm{BEC}$ research has been to extend the level of ultimate quantum control offered by a BEC to molecular systems. However, cooling molecules to the ultracold temperatures needed for BEC is especially challenging because of their complicated energy level structures. Standard laser cooling techniques that have thus far been developed and perfected for atoms are not suited for molecules since, in general, molecules lack the closed cycling transitions necessary to recycle the population during the cooling process. The added rotational degree of freedom of molecules is not the major hindrance to the existence of cycling transitions since rotational transitions are governed by strict selection rules. In fact, optical transitions can be found in a variety of molecules that are both electronically and rotationally closed [1]. The problem lies in the extensive vibrational structure of molecules. After undergoing spontaneous emission, a group of molecules that began in the same initial excited electronic state are usually spread over many vibrational levels in the electronic ground state potential because there is good Franck-Condon overlap between the initial state and numerous ground vibrational states.

Several unique methods for cooling molecules are currently being developed. Some groups are exploring new schemes to cool the molecules directly, while others rely on making cold molecules from a sample of already cooled atoms. Perhaps the most common route to creating cold molecules has been through photoassociation of cold atoms either in a MOT or in an atomic BEC. For example, a two-photon stimulated Raman transition in a ${ }^{87} \mathrm{Rb} \mathrm{BEC}$ has been used to produce ${ }^{87} \mathrm{Rb}_{2}$ diatomic molecules in a single rotational-vibrational state of the ground-state potential [2]. It is unclear whether the molecules possessed the coherence properties needed to consider them a $\mathrm{BEC}$ as the authors were not able to probe these properties. Also, ultracold sodium 
molecules have been produced via single-photon photoassiciation of a sodium BEC [3]. Other methods for making ultracold molecules include using a Helium buffer gas to cool paramagnetic molecules such as $\mathrm{CaH}$ in a magnetic trap [4] and using electrostatic beam slowing techniques on polar molecules such as $\mathrm{ND}_{3}$ [5].

Utilizing the natural atom-molecule coupling that arises from a Feshbach resonance is an alternate route for producing ultracold molecules from an atomic BEC and it is the one we follow here. Theory predicts coherent oscillations between the atomic and molecular states when the magnetic field is tuned in the vicinity of a Feshbach resonance $[11,12]$. To create a superposition of atoms and molecules and probe its coherence properties, we applied two rapid magnetic field pulses toward the Feshbach resonance that were separated by a free evolution time, $t_{\text {evolve }}$, in which the magnetic field was held at a constant value some distance from the resonance. The number of atoms in the condensate oscillated as a function of $t_{\text {evolve }}$ at a frequency corresponding to the energy splitting between atomic and molecular states during the free-evolution period, indicating that we coherently produced cold molecules from an atomic BEC. Whether this group of molecules could be considered a molecular condensate is up for discussion.

Our method of producing cold molecules by using fast magnetic field pulses near a Feshbach resonance may also have other applications. It may be possible to make composite bosons out of fermions by utilizing a Feshbach resonance in a cold Fermi gas [16]. Such diatomic molecules made of fermions may exhibit superfluid properties.

\section{Feshbach Physics}

The total energy of two colliding atoms is equal to the energy of a bound molecular state at a Feshbach resonance, and atom-molecule transitions can occur during collisions [6]. A schematic representation of the potentials involved is shown in the inset of Fig. 1. For our ${ }^{85} \mathrm{Rb}$ resonance, BEC atoms in the $F=2, m_{F}=-2$ state collide on the open-channel threshold. $F$ and $m_{F}$ are the total spin and spin-projection quantum numbers. The bound state in the closed channel differs in energy by an amount $\epsilon$ from the open-channel threshold. The bound molecular wave function can be described as a sum of amplitudes of different hyperfine components $\left(F, m_{F}\right)$ having $M_{F}=m_{F, 1}+m_{F, 2}=-4[7]$. Because of their different spin configurations, the atoms and molecules generally have different magnetic moments and the difference depends on magnetic field. Thus $\epsilon$ depends on magnetic field and the degree of atom-molecule coupling is magnetically tunable. The energy difference between the free atoms and the bound molecules versus magnetic field is plotted in Fig. 1.

Previous experiments have shown that inelastic losses are dramatically enhanced when the magnetic field is ramped across the Feshbach resonance [8, 9]. It is likely that the formation of molecules played a role in the loss, but there was no experimental evidence for the presence of molecules. More recently, we measured the time dependence of the loss by applying controlled magnetic-field pulses toward the Feshbach resonance [10]. We observed the surprising result that under some conditions, shorter, more rapid pulses actually led to more loss than longer, slower pulses that spent more time near the resonance. The time dependence of the loss was suggestive 


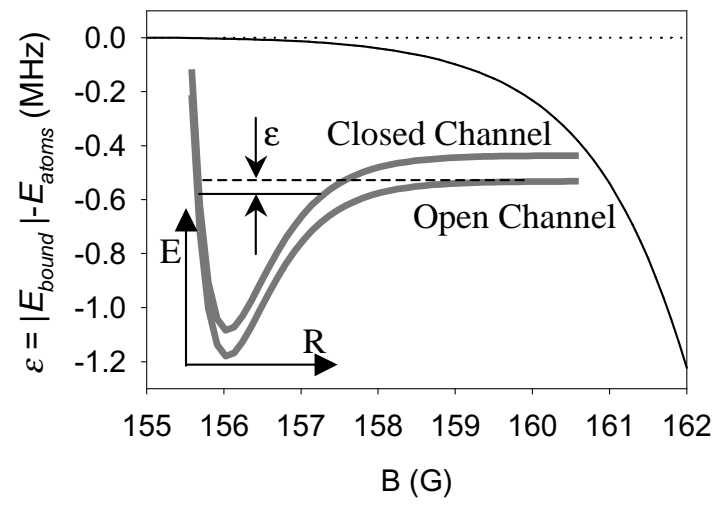

Figure 1: Feshbach resonance bound-state energy versus magnetic field. The solid curve is a theoretical coupled-channels calculation of the energy provided by S.J.J.M.F. Kokkelmans and C.H. Greene. The inset schematically shows the collision channels involved in the resonance. $\epsilon$ depends on magnetic field because the atoms and molecules have different magnetic moments and thus the potentials have different Zeeman shifts.

of a nonadiabatic mixing of states, with the only states within a reasonable energy range being the normal atomic BEC state and the nearby highest lying molecular state. Some simple modelling of the system using a Landau-Zener avoided crossing picture yielded results which qualitatively resembled the trends of our experimental data. Also, we observed Rabi-like oscillations in the number of atoms remaining after a single magnetic field pulse toward the resonance versus the length of the pulse. These oscillations were only visible over a very limited range of pulse heights ( $a$ near $1700 a_{0}$ ) because both a strong coupling and a condensate loss time that is long compared to the Rabi oscillation period were required.

\section{Experimental Methods}

The apparatus has been described in detail elsewhere $[9,10]$. We first created ${ }^{85} \mathrm{Rb}$ condensates typically containing 16,500 atoms, with fewer than 1,000 uncondensed thermal atoms. The initial number, $N_{\text {init }}$, fluctuated from shot to shot by $\sim 500$ atoms ( $\sim 3 \%$ number noise). After producing the condensate at a field of $\sim 162 \mathrm{G}$, we ramped the magnetic field adiabatically to $\sim 166 \mathrm{G}$, corresponding to an initial scattering length $a_{\text {init }} \simeq 10 a_{0}$, where $a_{0}=0.053 \mathrm{~nm}$. The spatial distribution of the atoms was Gaussian with a typical peak atom density of $n_{0}=5.4 \times 10^{13} \mathrm{~cm}^{-3}$. For some experiments where a lower initial density was desired, the adiabatic ramp to $\sim 166 \mathrm{G}$ was omitted so that the mean field repulsion at $\sim 162 \mathrm{G}\left(a_{\text {init }} \simeq 200 a_{0}\right)$ reduced the peak initial density to $n_{0}=1.1 \times 10^{13} \mathrm{~cm}^{-3}$. After preparing the condensate we applied a selected fast magnetic-field pulse sequence by sending an appropriate time-dependent current through an auxiliary magnetic-field coil [10]. A typical pulse 


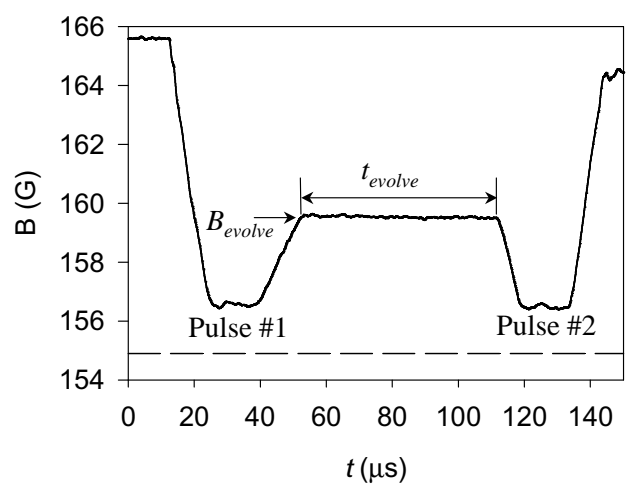

Figure 2: Typical magnetic field pulse shape. The two pulses correspond to a scattering length of $\sim 2500 a_{0}$. The dashed line indicates the position of the Feshbach resonance.

sequence is shown in Fig. 2. It is composed of two nearly identical short trapezoidal pulses separated by a region of constant magnetic field. Upon completion of the fastpulse sequence in Fig. 2, we ramped the magnetic field from $\sim 166 \mathrm{G}$ to $\sim 157 \mathrm{G}$ in $5 \mathrm{~ms}$ and held at that field for an additional $7 \mathrm{~ms}$ to allow the repulsive mean-field energy to expand the condensate. Then we turned off the magnetic trap and used destructive absorption imaging $12.8 \mathrm{~ms}$ later to observe the atomic condensate and measure the number of remaining atoms [13]. This detection scheme was neither sensitive to atoms with kinetic energies larger than $\sim 2 \mu \mathrm{K}$ nor to atoms in off-resonant molecular states. We determined the value of the magnetic field between the pulses by measuring the resonance frequency for transitions from the $F=2, m_{F}=-2$ to the $F=2, m_{F}=-1$ spin state by applying a $10 \mu \mathrm{s} R$ pulse to a trapped cloud of atoms and measuring loss of atoms from the trap [10].

\section{Burst and Remnant Oscillations}

After the application of a fast double magnetic field pulse sequence as described above there were two spatially distinct components visible in typical absorption images, as well as one undetected component. These three components were also observed in previous work after applying just a single pulse towards the resonance [10]. The most visible component was a cold BEC "remnant" whose spatial structure was similar to that of the initial condensate. This remnant of the condensate did not appear to be appreciably heated or excited by the fast-pulse sequence. The second detectable component was a comparatively hot $(\sim 150 \mathrm{nK})$ "burst" of atoms that remained trapped throughout the experiment and had a larger spatial extent than the remnant. The mean-field expansion energy that was imparted to the BEC remnant in order to measure its number was, as calculated using a variational approach [14], at most $\sim 3 \mathrm{nK}$. 

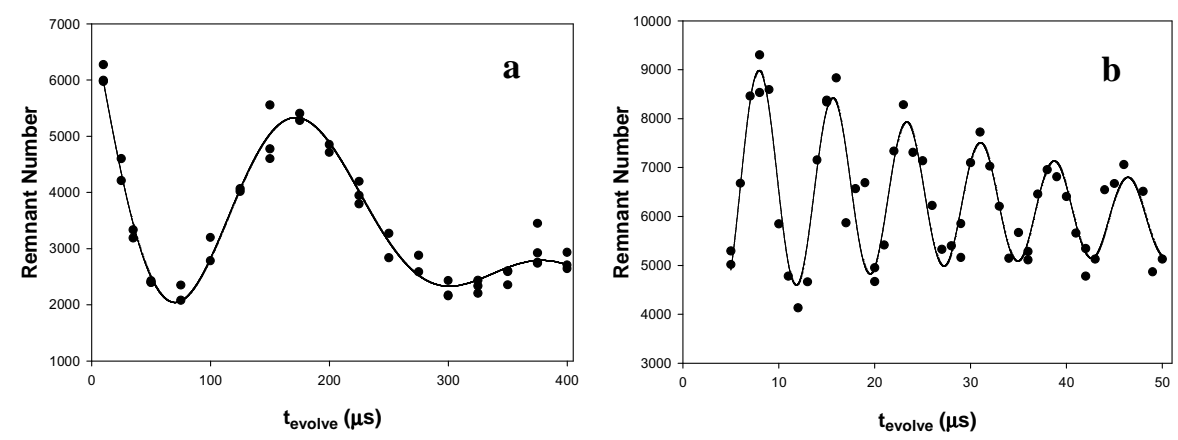

Figure 3: $N_{\text {remnant }}$ versus $t_{\text {evolve }}$. a, $B_{\text {evolve }}=156.133(6) \mathrm{G}\left(a_{\text {evolve }}=3565 a_{0}\right)$, $n_{0}=1.1 \times 10^{13} \mathrm{~cm}^{-3}$. Note that in this case, the two pulses reached $\sim 5000 a_{0}$. We have found that increasing the amplitude of the pulses above $\sim 2500 a_{0}$ to $\sim 20,000 a_{0}$ has no effect on the oscillations. The fit parameters are $\nu_{0}=4.5(6) \mathrm{kHz}, \tau=$ $174(13) \mu \mathrm{s}$, and $m=7.2(3)$ atoms $/ \mu \mathrm{s}$. b, $B_{\text {evolve }}=159.61(1) \mathrm{G}\left(a_{\text {evolve }}=600 a_{0}\right)$, $n_{0}=1.3 \times 10^{14} \mathrm{~cm}^{-3}$. Note that here $N_{\text {init }}=29,000$. The fit parameters are $\nu_{0}=$ $133(1) \mathrm{kHz}, \tau=38(8) \mu \mathrm{s}$, and $m=19(6)$ atoms $/ \mu \mathrm{s}$.

Finally, the "missing" component consisted of atoms that were in the initial sample but were not detected after the trap turn off.

In order to count the number of atoms in the remnant and burst effectively, we waited for the relatively dilute burst to be focused by the magnetic trap. We then fit the focused burst to a two-dimensional gaussian surface, excluding the central region that contained the remnant to obtain $N_{\text {burst }}$. This fit was subsequently subtracted from the original image and a pixel-by-pixel sum was performed over the central region to obtain $N_{\text {remnant }}$. $N_{\text {missing }}$ was calculated by subtracting $N_{\text {remnant }}$ and $N_{\text {burst }}$ from $N_{\text {init }}$.

$N_{\text {remnant }}$ versus $t_{\text {evolve }}$ is plotted in Fig. 3 for two different values of $B_{\text {evolve }}$. There is clearly an oscillation in $N_{\text {remnant }}$. The data has been fit to the function $y=$ $y_{0}+A \exp (-t / \tau) \sin (2 \pi \nu t+\phi)-m t$ to find the oscillation frequency $\nu=\sqrt{\nu_{0}^{2}-1 / \tau^{2}}$, decay time constant $\tau$, and a linear loss term $m$. We have taken similar data for a variety of different $B_{\text {evolve }}$ values. The measured frequencies are plotted versus $B_{\text {evolve }}$ in Fig. 4 along with theoretical predictions for the bound state energy relative to the atomic state. The bound state energy can be approximated by $\epsilon=-\hbar^{2} / m a^{2}$ when the scattering length is much larger than the radius of the interatomic potential, but a more sophisticated coupled-channels calculation is needed to determine the binding energy over the entire range. $\hbar$ is Plank's constant divided by $2 \pi, m$ is the atomic mass, and $a$ is the scattering length. The data agree well with the simple theoretical model over the range in which the theory is expected to be valid and with the coupledchannels result over the entire range of magnetic field.

$N_{\text {burst }}$ and the total number also oscillated at the same frequency as the remnant but with different phases as shown in Fig. 5. For conditions under which most data were collected, the burst contained an average of $\sim 5000$ atoms. $N_{\text {burst }}$ depended on 


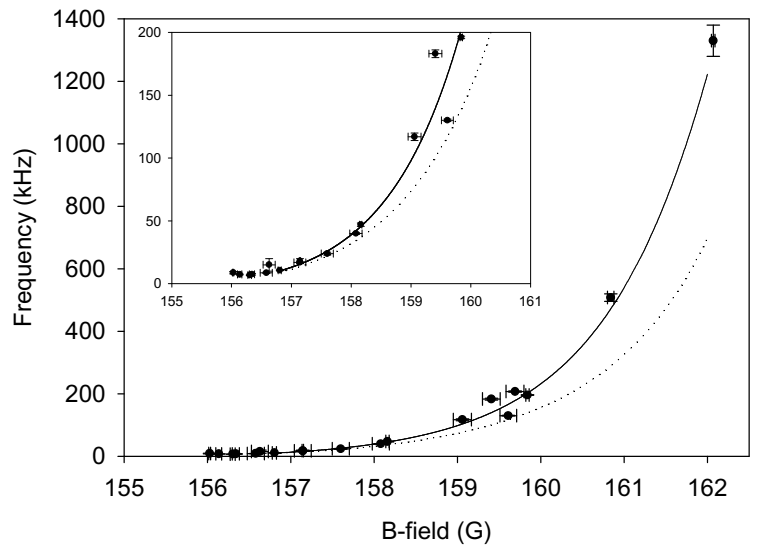

Figure 4: Oscillation frequency, $\nu$, versus magnetic field. The points are the measured frequencies. The solid line is the energy difference between the atom-atom threshold and the bound molecular state found by S. Kokkelmans with a coupled-channel scattering calculation. The dotted line is a plot of the simple theory prediction $\epsilon / h$. The inset is an expanded view of the lower-frequency data.

density and varied from half of the atoms lost from the condensate for our typical peak density of $n_{0}=5.4 \times 10^{13} \mathrm{~cm}^{-3}$ to nearly all of the atoms lost from the condensate for $n_{0}=1.1 \times 10^{13} \mathrm{~cm}^{-3}$.

The relative phase between the remnant and burst oscillations depended strongly on the fall time of the second pulse as shown in Fig. 6. This change in the relative phase between the two visible oscillating components affected the total number oscillation amplitude. As the fall time was increased, the burst and remnant oscillations became more in phase and their oscillations added more constructively to give a larger total number oscillation amplitude. As shown in the inset of Fig. 5, the missing atom fraction increased with the fall time of the second pulse and also levelled out near $150 \mu \mathrm{s}$.

The damping time for the oscillations, $\tau_{\text {decay }}$, was not measured with high precision for many values of $B_{\text {evolve }}$, leading to uncertainties as large as $100 \%$ for some fields. At frequencies near $200 \mathrm{kHz}$, we measured $\tau_{\text {decay }}=38(8) \mu \mathrm{s}$ for $n_{0}=5.4 \times 10^{13} \mathrm{~cm}^{-3}$ and $\tau_{\text {decay }}=91(33) \mu$ s when we decreased $n_{0}$ to $1.1 \times 10^{13} \mathrm{~cm}^{-3}$. There was no strong dependence of $\tau_{\text {decay }}$ on magnetic field except within $\sim 2 G$ of the Feshbach resonance where $\tau_{\text {decay }}$ decreased from $261(36) \mu$ s to $132(18) \mu$ s as the magnetic field decreased from 156.804(25) G towards resonance to $156.027(11) \mathrm{G}$ (for $n_{0}$ to $1.1 \times 10^{13} \mathrm{~cm}^{-3}$ ). The linear loss rate was on the order of 5 to 10 atoms $/ \mu$ s and preliminary data shows that there is no strong dependence on density or field, and the loss appears to be consistent with that due to a single pulse followed by a hold at $B_{\text {evolve }}$ for a time $t_{\text {evolve }}$ (no second pulse). The damping rate and linear loss slope are subjects of continuing investigations. 


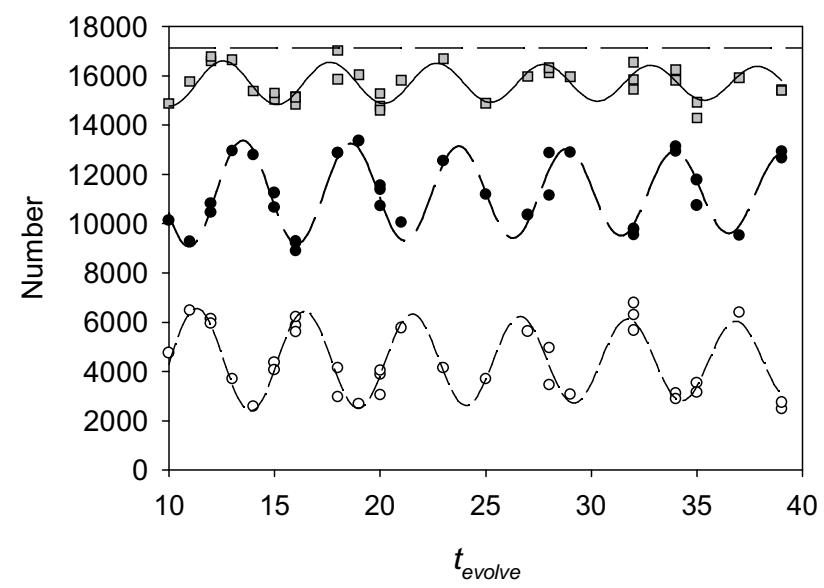

Figure 5: Number versus $t_{\text {evolve }}$ for $n_{0}=1.1 \times 10^{13} \mathrm{~cm}^{-3}$. From bottom to top, there is $N_{\text {burst }}$ (open circles), $N_{\text {remnant }}$ (filled circles), and the total number of observed atoms (gray squares). Each data set was fitted as explained in the text. $N_{\text {init }}=$ 17,100 is indicated by the flat dashed line. $B_{\text {evolve }}=159.84(2) \mathrm{G}$ and the remnant data fitted to an oscillation frequency of $196(1) \mathrm{kHz}$ and $\tau_{\text {decay }}=91(33) \mu \mathrm{s}$. Not all data used to obtain $\tau_{\text {decay }}$ are shown.

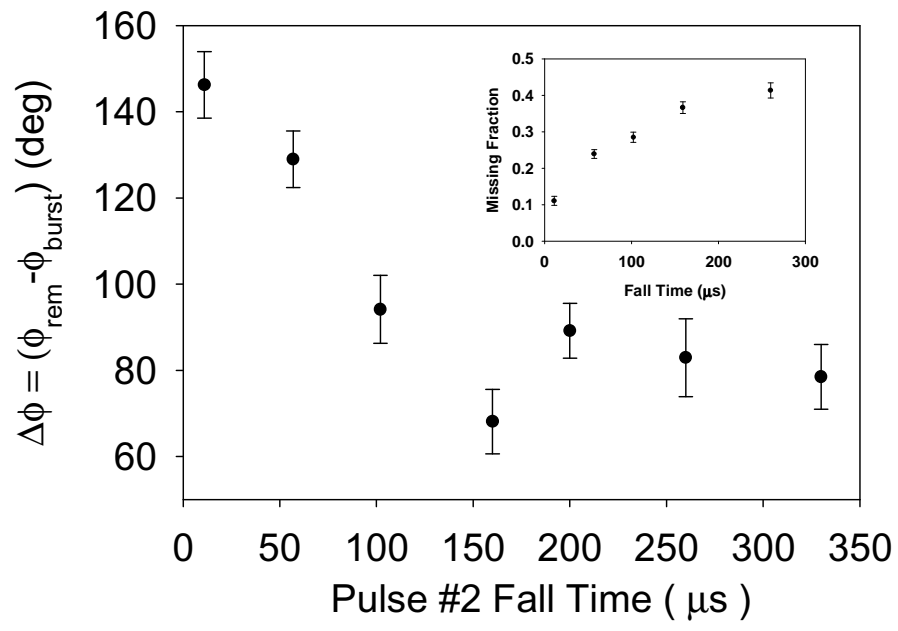

Figure 6: Phase difference between remnant and burst oscillations versus fall time of the second pulse. Measurements were done with $n_{0}=1.1 \times 10^{13} \mathrm{~cm}^{-3}$ at an oscillation frequency around $200 \mathrm{kHz}$. The inset shows the missing atom fraction as a function of the fall time of the second pulse. 
Our interpretation of our observations is that the first magnetic field pulse coherently transfers some atoms in the condensate to the molecular state. The superposition then evolves in accordance with the energy splitting between the states. The second pulse mixes the atom and molecule states again such that the final state depends on the relative phase between the atomic and molecular fields at the time of the second pulse. The mechanism of the burst is yet unknown, as is the fate of the missing atoms. Can the molecules be considered a molecular condensate? A beyond mean-field pairing theory proposed by S.J.J.M.F Kokkelmans and M.J. Holland [15] has been successful in predicting the existence of the these three oscillating components and the burst energies roughly agree with our experimental data. The major discrepancy is that this theory predicts far fewer missing atoms than are observed, particularly for higher initial densities. Also, the behavior of the system when the fall time of the second pulse was varied could not be predicted using this theory.

\section{References}

[1] G. Meijer ChemPhysChem 3, 495-501 (2002).

[2] R.H. Wynar, R.S. Freeland, D.J. Han, C. Ryu, and D.J. Heinzen Science 287, 1016-1019 (2000).

[3] C. McKenzie et al. Phys. Rev. Lett. 88, 120403/1-4 (2001).

[4] J.D. Weinstein et al. Nature 395, 148-150 (1998).

[5] H.L. Bethlem et al. Nature 406, 491-493 (2000).

[6] A.J. Moerdijk, B.J. Verhaar, and A. Axelson Phys. Rev. A 51, 4852 (1995).

[7] F.A. van Abeelen, D.J. Heinzen, and B.J. Verhaar Phys. Rev. A 57, R4102-R4105 (1998).

[8] J. Stenger et al. Phys. Rev. Lett. 82, 2422 (1999).

[9] S.L. Cornish et al. Phys. Rev. Lett. 85, 1795 (2000).

[10] N.R. Claussen et al. Phys. Rev. Lett. 89, 010401/1-4 (2002).

[11] E. Timmermans, P. Tommasini, M. Hussein, and A. Kerman Phys. Rep. 315, 199 (1999)

[12] P.D. Drummond, K.V. Kheruntsyan, and H. He Phys. Rev. Lett. 81, 3055 (1998).

[13] J.L. Roberts et al. Phys. Rev. Lett. 86, 4211 (2001).

[14] V.M. Pérez-García et al. Phys. Rev. A 56, 1424 (1997).

[15] S.J.J.M.F. Kokkelmans, and M.J. Holland preprint cond-mat/0204504 at http://xxx.lanl.gov (2002).

[16] M. Haw Nature 417, 892 (2002). 